\title{
miR-22 and cerebral microbleeds in brainstem and deep area are associated with depression one month after ischemic stroke
}

\author{
Jia Hu(i) ${ }^{1,2}$, Wei Zhou (i) ${ }^{3}$, Zhiming Zhou (i) ${ }^{2}$, Qian Yang (i) ${ }^{2}$, Junfeng Xu(i) ${ }^{2}$, and Wanli Dong (i) ${ }^{1}$ \\ ${ }^{1}$ Department of Neurology, The First Affiliated Hospital of Soochow University, Suzhou, Jiangsu, China \\ ${ }^{2}$ Department of Neurology, The First Affiliated Hospital, Yijishan Hospital of Wannan Medical College, Wuhu, Anhui, China \\ ${ }^{3}$ Department of Cardiovascular surgery, The First Affiliated Hospital, Yijishan Hospital of Wannan Medical College, \\ Wuhu, Anhui, China
}

\begin{abstract}
In this study, we aimed to explore the relationship among miR-22, deep cerebral microbleeds (CMBs), and post-stroke depression (PSD) 1 month after ischemic stroke. We consecutively recruited 257 patients with first-ever and recurrent acute cerebral infarction and performed PSD diagnosis in accordance with the Diagnostic and Statistical Manual IV criteria for depression. Clinical information, assessments of stroke severity, and imaging data were recorded on admission. We further detected plasma miR-22 using quantitative PCR and analyzed the relationship among miR-22, clinical data, and PSD using SPSS 23.0 software. Logistic regression showed that deep (OR=1.845, 95\% $\mathrm{Cl}: 1.006-3.386, \mathrm{P}=0.047)$ and brain stem CMBs $(\mathrm{OR}=2.652,95 \% \mathrm{Cl}: 1.110-6.921, \mathrm{P}=0.040)$, as well as plasma miR-22 levels $(\mathrm{OR}=2.094,95 \% \mathrm{Cl}: 1.066-4.115, \mathrm{P}=0.032)$ were independent risk factors for PSD. In addition, there were significant differences in baseline National Institutes of Health Stroke Scale scores (OR=1.881, 95\%Cl: 1.180-3.011, $\mathrm{P}=0.007)$ and Widowhood scores (OR=1.903, 95\%Cl: 1.182-3.063, $\mathrm{P}=0.012)$. Analysis of the receiver operating curve $(A \cup C=0.723,95 \% \mathrm{Cl}: 0.562-0.883, P=0.016)$ revealed that miR-22 could predict $P S D$ one month after ischemic stroke. Furthermore, plasma miR-22 levels in brainstem and deep CMBs patients showed an upward trend $(P=0.028)$ relative to the others. Patients with acute ischemic stroke, having brainstem and deep cerebral microbleeds, or a higher plasma miR-22 were more likely to develop PSD. These findings indicate that miR-22 might be involved in cerebral microvascular impairment and post-stroke depression.
\end{abstract}

Key words: Depression; microRNA; Stroke; Cerebral microbleeds; Magnetic resonance imaging

\section{Introduction}

Post-stroke depression (PSD) is an emotional disorder and one of the most common complications of ischemic stroke. The occurrence of PSD is always followed by negative clinical outcomes including cognitive and functional impairment. Furthermore, the condition is a prominent barrier to stroke rehabilitation, contributing to reduced quality of life and increased risk of stroke and mortality (1). Given that its pathogenesis is still unclear, there is a need to study risk factors related to PSD and further explore new stable biomarkers for its diagnosis and treatment.

MicroRNAs (miRNAs) are small non-coding RNAs of about 19-25 nucleotides long that bind onto mRNA $3^{\prime}$ untranslated region (UTR) and regulate mRNA degradation as well as inhibit protein synthesis (2). Previous studies have reported that miRNAs are involved in regulating endothelial dysfunction, cell proliferation and apoptosis, inflammatory response, oxidative stress, angiogenesis, and neurogenesis (3). At the same time, they play an important role in release of neurotransmitters and synaptic plasticity (4). Moreover, these RNAs have been shown to play a critical role in major depression and suicidal behavior, generating a negative outcome (5). Circulating miRNAs have been identified as novel potential blood biomarkers in some mental diseases including major depression. Previous studies have shown that differential expression of miRNAs influenced homeostasis of neural and synaptic pathways, by negatively regulating expression of genes such as brainderived neurotrophic factor ( $B D N F)$-tyrosine kinase receptor $\mathrm{B}$ (TrkB) pathways and other important signaling pathways $(6,7)$. Our previous research showed that tyrosine kinase receptor $B$ was associated with PSD in Chinese people (8).

A search through miRNAs target genes online prediction software for miRNAs related to $\operatorname{TrkB}$ revealed that miR-22 is strongly associated with NTRK2. Meanwhile,

Correspondence: Wanli Dong: <dwl1177@163.com>

Received July 30, 2019 | Accepted February 26, 2020 
previous research using gene chip has shown that miR-22 is just one of 25 differentially expressed miRNAs between PSD and non-PSD patients (9), although its diagnostic efficacy and possible mechanisms of action remain unknown. miR-22 has also been reported to be involved in pathophysiological processes such as endothelial dysfunction. Overexpressing miR-22 was found to induce inflammation, damage endothelial cells, increase vascular permeability, and finally destroy the blood-brain barrier (10). Dysfunction of endothelial cells and destruction of the blood-brain barrier were involved in the pathophysiology of microbleeds (11). In the current study, we hypothesized that cerebral micro-vascular impairment might be one of the targets of miR-22 driving PSD.

To our knowledge, this is the first study to demonstrate a relationship between post-stroke depression and miR22 , as well as its clinical indicators. The aim of the study, therefore, was to clarify the role of miR-22 in the pathogenesis of PSD. We also sought to confirm whether miR-22 can serve as a novel biomarker and a potential therapeutic target of PSD.

\section{Material and Methods}

\section{Study subjects}

This study enrolled 351 patients diagnosed with acute cerebral infarction following magnetic resonance imaging (MRI) scans performed at the Department of Neurology, Yijishan Hospital from March 2015 to September 2017. Inclusion criteria were as follows: i) all enrolled patients met the criteria defined by the World Health Organization Multinational Monitoring of Trends and Determinants in Cardiovascular Disease (WHO-MONICA) for acute ischemic stroke (AIS), and were verified by MRI within $24 \mathrm{~h}$ of admission; ii) the course of disease was less than 1 week; and iii) patients were over 18 years of age. Exclusion criteria included the following: i) patients with psychiatric conditions such as depression; ii) patients with unconsciousness, dementia, or significant cognitive impairment; iii) patients with severe aphasia or dysarthria, visual or auditory impairment; iv) patients with metabolic abnormalities, tumors, significant acute inflammatory disease, or other medical illness besides stroke; and v) patients on whom MRI scans were not performed for various reasons. All patients were followed up for 1 month. A total of 257 patients completed the follow-up and were eventually enrolled in the study. In addition, the study ensured that each patient's clinical symptoms met the diagnostic criteria for cognitive impairment and dementia outlined in the "Chinese Guidelines for Diagnosis and Treatment of Cognitive Impairment and Dementia" (http://www. chinadoi.cn/portal $/ \mathrm{mr}$.action?doi=10.3760/cma.j.issn.03762491.2010.41.003). Patients with MMSE $\leqslant 9$ were considered to have severe cognitive impairment or dementia.

The study was approved by the Ethics Committee of First Affiliated Yijishan Hospital of Wannan Medical
College. Written informed consents were signed by participants or their relatives before including them in the study.

\section{Methods}

Collection of clinical data. Baseline clinical data collected from the enrolled subjects included demography (age, sex, body mass index, and education), vascular risk factors (hypertension, diabetes mellitus, hyperlipidemia, and coronary heart disease), as well as medical history (smoking, alcohol consume, and cases of previous stroke). Stroke subtypes were classified according to criteria outlined by TOAST (12) while its severity was evaluated by trained neurologists using the National Institutes of Health Stroke scale (NIHSS) within $24 \mathrm{~h}$ of admission. The Hamilton Depression-17 (HAMD-17) scale (13) and clinical interviews were performed by a trained neurologist one month after onset of stroke (Qian Yang). The HAMD-17 scale was also used to assess depressive symptoms one month after onset while diagnosis of PSD was done in accordance with Diagnostic and Statistical Manual IV criteria for depression one month after stroke (14).

\section{Assessment of imaging data}

Head MRI scans (3.0t GE Multisync LCD, USA) were performed within 1 week of admission. Briefly, neuroimaging markers of small vessel disease were assessed using STRIVE nomenclature by analyzing MR images. Scan sequence included T1 and T2 weighted imaging, sequence of fluid attenuated inversion recovery (FLAIR), diffusion weighted imaging (DWI), magnetic resonance angiography (MRA), and susceptibility-weighted imaging (SWI). The parameters used included 3.0t MRI SWI: TR $76.1 \mathrm{~ms}$, TE $42.58 \mathrm{~ms}$, slice thickness/gap 2/1 mm, 3.0t DWI TR $3000 \mathrm{~ms}$, TE $65.5 \mathrm{~ms}$, slice thickness/gap 6/7 mm; MRA TR $21 \mathrm{~ms}$, TE $2.5 \mathrm{~ms}$, and slice thickness/ gap $1.4 / 0.7 \mathrm{~mm}$. Based on the STRIVE nomenclature (15), imaging data was divided into the following aspects: enlarged perivascular spaces, white matter hyperintensities, cerebral microbleeds, lacunar cerebral infarction, and recent small subcortical infarction.

Cerebral microbleeds (CMB) were defined as multiple ovoid foci with marked loss of signal intensity on T2*weighted, gradient-recalled echo MRI. SWI showed low round point signal with a diameter of $2 \sim 10 \mathrm{~mm}$, at least half of which was surrounded by brain parenchyma with no edema. Symmetrical basal ganglia calcification, vascular cavitation effect, cavernous hemangioma, and diffuse axonal injury were excluded. Lesions located within the infarct area were not considered microbleeds. In addition, CMBs were divided into lobar, deep, and posterior fossa (brain stem and cerebellum) groups while white matter hyperintensities on MRI were defined as hyperintensities $\geqslant 5 \mathrm{~mm}$ on T2 and T2FLAIR images. Severity of white matter hyperintensities was assessed using the Fazekas 
scale (0-3 points). White matter hyperintensities (WMHs) were divided into periventricular (PVWMHs) and deep (DWMHs). Meanwhile, MRI was used to locate infarction lesions and count the number of acute infarctions in the cortex, subcortical white matter, deep, and sub-tentorial.

\section{Selection and detection of miRNAs}

According to our previous research, tyrosine kinase receptor $B$ is strongly associated with PSD in Chinese people. In this study, we searched for miRNAs related to the TrkB gene (NTRK2, gene number: ENSG0000014 8053) in TargetScan; an online software for prediction of microRNA target genes (http://www.targetscan.org/) and miRBD (http://mirbd.org/miRBD/Index.html). An intersection between results from the two databases showed that miR-22 was most strongly related to NTRK2. At the same time, we compared this with previous results of miRNAs gene chip in post-stroke depression (9) and found that miR-22 was one of 25 miRNAs that showed a significant differential expression in patients with post-stroke and non-post-stroke depression.

In both groups, we selected 20 (from a total of 40 patients) for each group for detection of plasma miR22 . We ensured that there were no significant differences in gender, age, stroke site, NIHSS score, and TOAST type between the two groups $(P>0.05)$.

In the morning of the day after admission, $4 \mathrm{~mL}$ of venous blood was collected into an EDTA anticoagulant tube. The blood sample was centrifuged at $800 \mathrm{~g}$ for $15 \mathrm{~min}$ at $4^{\circ} \mathrm{C}$ within $2 \mathrm{~h}$ of collection, the upper plasma was encapsulated in EP tubes, and then frozen at $-80^{\circ} \mathrm{C}$ before measurement. Total plasma RNA was isolated and purified using the mirVanaTM RNA Isolation kit (Applied Biosystem p/n AM1556, USA). Concentration of each total RNA sample was determined using a Nanodrop 2000 spectrophotometer (Thermo, USA). Each sample was tested 3 times and those with an A260/A280 value between 1.9 and 2.2 were used in subsequent experiments. RNA was reverse transcribed using the miScript reverse transcription kit (Qiagen; 218161, Germany) according to the manufacturer's instructions. A synthetic C. elegans miR-39 (cel-miR-39, Qiagen; 219610), which is devoid of sequence homology to human microRNAs, was included into the plasma after lysis as an external reference. Primers were designed using Primer 5.0 software according to GenBank and miRBASE databases (Table 1), then synthesized by Shanghai Shengong Biotechnology Co. Ltd. (China). To determine expression of the miRNAs, we performed a real-time PCR using the miScript SYBR Green PCR kit (Qiagen; 218073) according to the manufacturer's instructions and each PCR was repeated 3 times. Melting curves were analyzed using the software in the fluorescence quantitative PCR machine and $\mathrm{Ct}$-values generated. Calculation of miR-22 expression was conducted by $2^{-\triangle \triangle \mathrm{Ct}}$ relative quantification method, using cel-miR39 as the internal reference gene (16).
Table 1. Primer sequences for quantitative real time polymerase chain reaction reverse transcription.

\begin{tabular}{lc}
\hline Primer & Sequence $\left(5^{\prime}\right.$ to $\left.3^{\prime}\right)$ \\
\hline qPCR-miR-22-F & TGCGGAGTTCTTCAGTGGCAA \\
qPCR-miR-22-R & CAGTGCAGGGTCCGAGGT \\
qPCR- Cel-miRNA-39-F & CGTCGATCACCGGGTGTAAA \\
qPCR- Cel-miRNA-39-R & CTCTGTCTCTCGTCTTGTTGGTAT \\
\hline
\end{tabular}

\section{Data analysis}

SPSS version 23.0 statistical software for data processing and analysis was used. The KolmogorovSmirnov test was first used for evaluating normality of the data. Data showing a normal distribution are reported as means $\pm S D$, with an independent sample $t$-test used for comparison between groups. Data with non-normal distribution are reported as median and interquartile range. Comparisons between groups were conducted by the Mann-Whitney U-test, while comparisons among multiple groups were done using the Kruskal-Wallis test. Count data are reported as frequencies and percentages. A multiple logistic regression analysis was used to evaluate PSD-related factors by calculating odds ratio (OR) and 95\% confidence interval $(\mathrm{Cl})$. A Pearson correlation was used to generate a relationship among variables. Finally, the receiver operating curve (ROC) was drawn to analyze the diagnostic efficacy of miR-22 at $P \leqslant 0.05$ significance level.

\section{Results}

\section{General patient data}

Of the 257 patients enrolled in the study, 151 (58.8\%) were male and $106(41.2 \%)$ were female. The average age of these patients was $62.3 \pm 10.7$ years (Figure 1). Excluded patients $(n=436)$ had no significant difference in gender (48.4 vs $41.2 \%, \mathrm{P}=0.068$ ) relative to the enrolled patients $(n=257)$. On the other hand, significant differences were noted in age $(64.8 \pm 11.5$ vs $62.3 \pm 10.7$, $\mathrm{P}=0.005$ ) and severity of stroke (NIHSS score $6.5 \pm 5.3 \mathrm{vs}$ $5.1 \pm 4.2, P<0.001)$. In the enrolled group, depression was noted in $73(28.4 \%)$ of the patients while the other $184(71.6 \%)$ showed no symptom one month after stroke. Basic characteristics of the 257 patients with and without post-stroke depression are reported in Table 2. In summary, patients in the PSD and non-PSD groups showed no difference with regards to age, gender, history of stroke, common vascular risk factors of ischemic stroke (such as hypertension, hyperlipidemia, diabetes, coronary heart disease, smoking, and drinking) as well as TOAST classification of stroke etiology. On the other hand, significant differences were recorded in the groups with regards to education $(P=0.027)$, widowhood $(P=0.002)$, BMl levels $(P=0.033)$, and baseline NIHSS scores $P<0.001)$. 


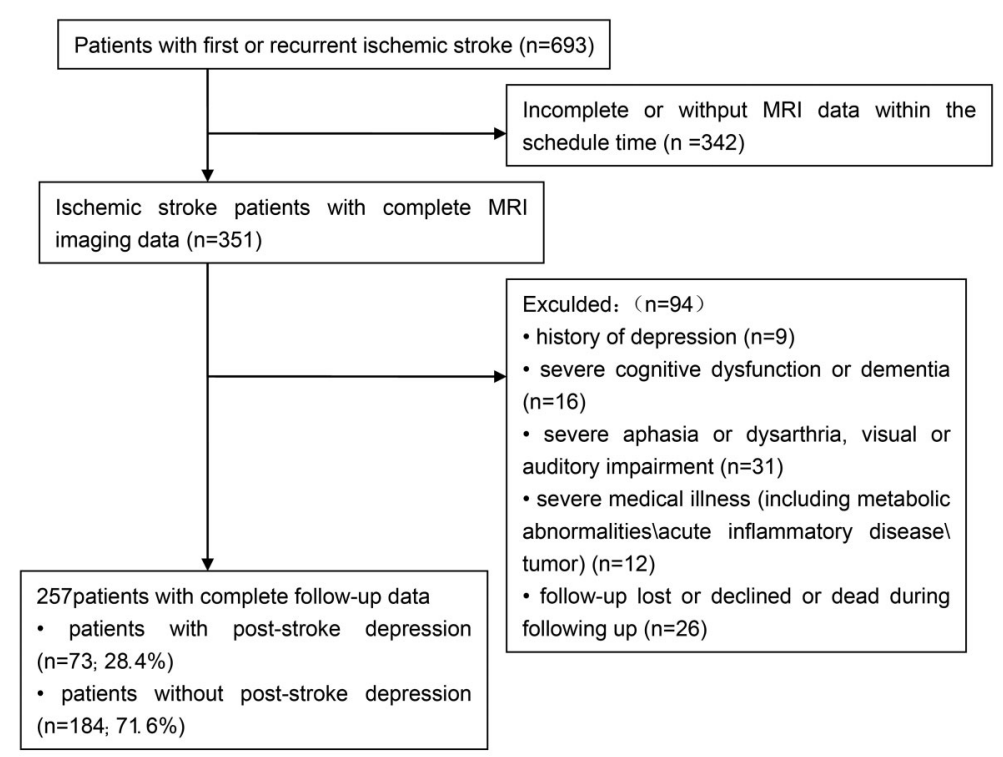

Figure 1. Study recruitment profile.

Table 2. Baseline clinical characteristics of patients with and without post-stroke depression (PSD) at 1 month.

\begin{tabular}{lccrc}
\hline Variables & $\begin{array}{c}\text { PSD group } \\
(\mathrm{n}=73)\end{array}$ & $\begin{array}{c}\text { Non-PSD group } \\
(\mathrm{n}=184)\end{array}$ & $\mathrm{t} / \mathrm{Z} / \mathrm{X}^{2}$ & $\mathrm{P}$ value \\
\hline Demographic characteristics & & & & \\
Age $(\mathrm{y}$, mean $\pm \mathrm{SD})$ & $62.7 \pm 10.1$ & $62.1 \pm 10.3$ & 0.423 & 0.672 \\
Female $(\mathrm{n}, \%)$ & $34(46.60)$ & $72(39.1)$ & 1.195 & 0.274 \\
Educational (y, mean $\pm \mathrm{SD})$ & $4.6 \pm 3.4$ & $5.6 \pm 3.2$ & -2.219 & 0.027 \\
BMI (kg/m ${ }^{2}$, mean \pm SD) & $24.9 \pm 2.5$ & $24.2 \pm 2.3$ & 2.146 & 0.033 \\
Widowhood (n, \%) & $24(32.9)$ & $29(15.8)$ & 9.353 & 0.002 \\
Previous stroke (n, \%) & $19(26.0)$ & $40(21.7)$ & 0.543 & 0.461 \\
Vascular risk factors (n, \%) & & & & \\
Hypertension & $61(83.6)$ & $134(72.8)$ & 3.291 & 0.069 \\
Hyperlipidemia & $31(42.5)$ & $71(38.6)$ & 0.329 & 0.567 \\
Diabetes mellitus & $32(43.8)$ & $73(39.7)$ & 0.345 & 0.541 \\
Coronary heart disease & $14(19.2)$ & $25(13.6)$ & 1.269 & 0.260 \\
Active smokers & $30(41.1)$ & $72(39.1)$ & 1.146 & 0.285 \\
Alcohol consumption & $21(28.8)$ & $44(23.9)$ & 0.420 & 0.652 \\
Type of stroke etiology (n, \%) & & & 0.268 & 0.605 \\
Atherothrombotic & $24(32.9)$ & $61(33.2)$ & & \\
Lacunar & $16(21.9)$ & $33(17.9)$ & & \\
Cardioembolic & $18(24.7)$ & $43(23.4)$ & & \\
Others & $15(20.5)$ & $47(25.5)$ & & \\
Baseline NIHSS score (median, IQR) & $9(7-12)$ & $8(6-11)$ & 7.321 & 0.001 \\
\hline
\end{tabular}

BMI: body mass index; NIHSS: National Institutes of Health Stroke Scale; IQR: interquartile range; SD: standard deviation. Data were analyzed by the $t$-test, Mann-Whitney U-test, or chi-square test. 
Table 3. Imagining characteristics in patients with and without post-stroke depression (PSD) at 1 month.

\begin{tabular}{|c|c|c|c|c|}
\hline Variate & $\begin{array}{l}\text { PSD group } \\
\quad(n=73)\end{array}$ & $\begin{array}{l}\text { Non-PSD group } \\
\quad(n=184)\end{array}$ & $t / Z / X^{2}$ & $P$ value \\
\hline EPVS (median, IQR) & $1(1-2)$ & $1(0-2)$ & 0.847 & 0.380 \\
\hline Fazekas DWMHs score (median, IQR) & $1(1-2)$ & $1(1-2)$ & 0.738 & 0.460 \\
\hline Fazekas PVWMHs score (median, IQR) & $1(1-1)$ & $1(0-1)$ & 0.981 & 0.327 \\
\hline Number of CMBs (mean $\pm \mathrm{SD}$ ) & $9.5 \pm 6.1$ & $9.7 \pm 4.8$ & -0.255 & 0.799 \\
\hline \multicolumn{5}{|l|}{ Location of CMBs $(n, \%)$} \\
\hline Lobar & $27(37.0)$ & $64(34.8)$ & 0.111 & 0.739 \\
\hline Deep & $21(28.8)$ & $31(16.8)$ & 4.601 & 0.032 \\
\hline Brainstem & $13(17.8)$ & $14(7.6)$ & & 0.023 \\
\hline Cerebellum & $3(4.1)$ & $12(6.5)$ & & 0.566 \\
\hline Lacure (n, \%) & $21(28.8)$ & $41(22.3)$ & 1.201 & 0.273 \\
\hline $\begin{array}{l}\text { Number of acute ischemic cerebral } \\
\text { infarction (median, IQR) }\end{array}$ & $1(1-1)$ & $1(1-1)$ & -0.323 & 0.747 \\
\hline Location of acute infarct (n, \%) & & & 0.210 & 0.647 \\
\hline Cortical & $17(23.3)$ & $42(22.8)$ & & \\
\hline Subcortical white matter & $7(9.6)$ & $20(10.9)$ & & \\
\hline Deep & $28(38.4)$ & $81(44.0)$ & & \\
\hline Infra tentorial & $21(28.8)$ & $41(22.3)$ & & \\
\hline Stroke characteristics (\%) & & & 1.262 & 0.261 \\
\hline Small infarct $(<15 \mathrm{~mm})$ & 25 & 77 & & \\
\hline Large infarct $(\geqslant 15 \mathrm{~mm})$ & 48 & 107 & & \\
\hline Lateralization (n, \%) & & & 0.938 & 0.333 \\
\hline Left hemisphere & $29(39.7)$ & $83(45.1)$ & & \\
\hline Right hemisphere & $32(43.8)$ & $78(42.4)$ & & \\
\hline Bilateral hemisphere & $12(16.4)$ & $23(12.5)$ & & \\
\hline
\end{tabular}

EPVS: enlarged perivascular spaces; DWMHs: deep white matter hyperintensities; PVWMHs: periventricular white matter hyperintensities; CMBs: cerebral microbleeds; AIS: acute ischemic stroke; IQR: interquartile range; SD: standard deviation. Data were analyzed by the $t$-test, Mann-Whitney U-test, Kruskal-Wallis test, or chi-square test.

Brain stem and deep CMBs were associated with PSD Patients in the PSD group were more likely to have brain stem (17.8 vs 7.6\%; $\mathrm{P}=0.023)$ and deep CMBs (28.8 vs $16.8 \% ; P=0.032$ ) compared to those in the non-PSD group (Table 3). We found no significant difference between PSD and the non-PSD groups with regards to Fazekas PVWMHs and Fazekas DWMHs scores, CMBs in other parts of the brain except frontal lobe, brainstem, and deep area, as well as the number, size, location, or laterality of acute infarction lesions.

We incorporated levels of the biomarker miR-22 into a logistic regression and performed an adjusted logistic regression. Results showed that deep $(\mathrm{OR}=1.845,95 \% \mathrm{Cl}$ : 1.006-3.386, $\mathrm{P}=0.047)$ and brain stem $\mathrm{CMBs}(\mathrm{OR}=2.652$, 95\% $\mathrm{Cl}$ : $1.110-6.921, \mathrm{P}=0.040)$ were significant predictors. In addition, the plasma level of miR-22 (OR=2.094, 95\% $\mathrm{Cl}: 1.066-4.115, \mathrm{P}=0.032$ ) was statistically related to PSD. Other predictors included baseline NIHSS (OR= 1.881, 95\% Cl: $1.180-3.011, \mathrm{P}=0.007)$ and widowhood (OR=1.903, 95\%Cl: 1.182-3.063, $\mathrm{P}=0.012$ ) (Table 4).
Table 4. Multivariate logistic model of the clinical determinants of post-stroke depression.

\begin{tabular}{lcc}
\hline Variables & OR $(95 \% \mathrm{Cl})$ & $\mathrm{P}$ value \\
\hline Brainstem CMBs & $2.652(1.110-6.921)$ & 0.040 \\
Deep CMBs & $1.845(1.006-3.386)$ & 0.047 \\
Years of educational & $0.867(0.669-1.124)$ & 0.282 \\
BMI & $1.432(0.987-2.061)$ & 0.058 \\
Widowhood & $1.903(1.182-3.063)$ & 0.012 \\
Baseline NIHSS score & $1.881(1.180-3.011)$ & 0.007 \\
Plasma miR-22 level & $2.094(1.066-4.115)$ & 0.032 \\
\hline
\end{tabular}

CMBs: cerebral microbleeds; BMI: body mass index; NIHSS: National Institutes of Health Stroke Scale.

\section{Correlation between plasma miR-22 expression and post-stroke depression}

Analysis of plasma miR-22 level showed an upward trend in subjects in the PSD group relative to those in the non-PSD group $(\mathrm{P}<0.0001)$ (Figure 2$)$. 


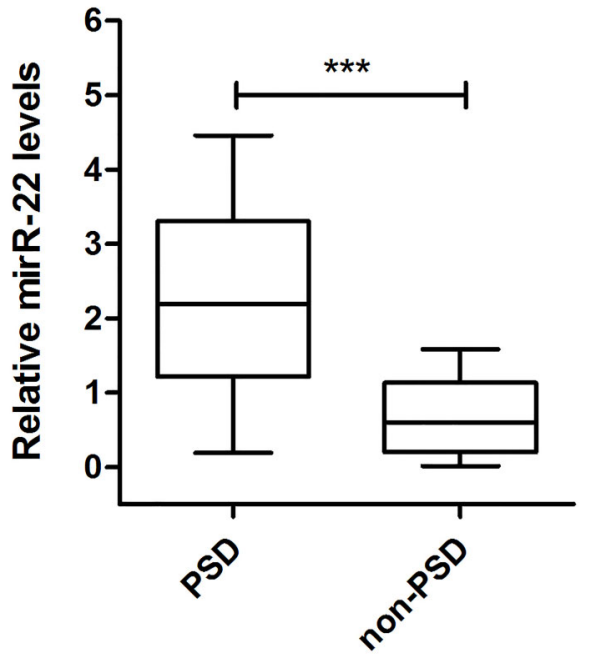

Figure 2. Relative expression of miR-22 in plasma compared between the post-stroke depression (PSD) group and the nonPSD group. Data are reported as median and interquartile range. ${ }^{* * * P}<0.001$ (Mann-Whitney U-test).

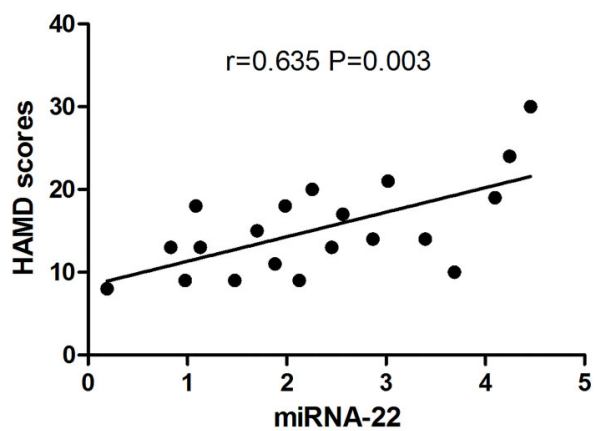

Figure 3. Pearson's correlation analysis between the relative expression of plasma miR-22 and Hamilton depression (HAMD) score in post-stroke depression patients.

Similarly, the Pearson's test showed that HAMD score was positively correlated with plasma miR-22 in post-stroke depression one month after stroke $(r=0.635, P=0.003)$ (Figure 3).

Meanwhile, ROC curves showed that miR-22 could predict PSD one month after ischemic stroke. Specifically, an AUC of 0.723 (95\% Cl: 0.562-0.883, $\mathrm{P}=0.016)$, with a cut-off point of $0.90,50 \%$ specificity, and $90 \%$ sensitivity were recorded (Figure 4). Plasma miR-22 concentrations above 0.90 at baseline represented a high risk of $P S D$.

A further analysis of the relationship between miR22 and brainstem and deep CMBs showed an upward trend of plasma miR-22 in patients with brainstem and deep CMBs compared to those without $(P=0.028)$ (Figure 5).

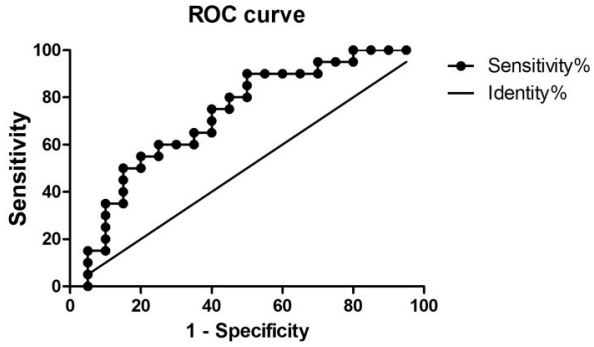

Figure 4. Receiver operating characteristic (ROC) curve demonstrating the predictive value of the relative expression of plasma miR-22 in post-stroke depression within 1 month.

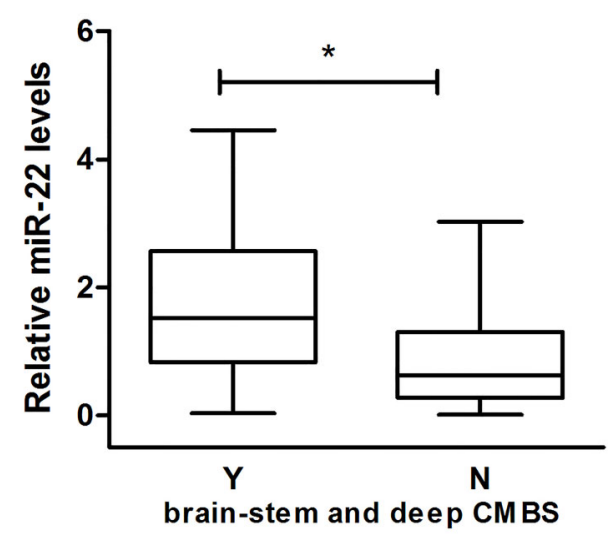

Figure 5. Boxplots comparison of the relative expression of miR22 in patients with $(\mathrm{Y})$ and without $(\mathrm{N})$ cerebral microbleeds (CMBs) in brainstem and deep area. Data are reported as median and interquartile range. ${ }^{*} \mathrm{P}<0.05$ (Mann-Whitney U-test).

\section{Discussion}

The findings of this study showed that acute ischemic stroke patients with brainstem and deep CMBs or a higher plasma miR-22 level were more likely to develop PSD. Specifically, we found that brain stem and deep CMBs were independent predictors of PSD. In addition, plasma miR-22 was related to PSD while miR-22 might be a new biomarker for the condition one month after stroke; taken together, these findings provided a new prospect for diagnosis and therapy of PSD.

CMBs, a type of cerebral vascular disease, have been implicated in the damage of early endothelial cells resulting in damage of the blood-brain barrier, neuroinflammation, and impairment of immune function (11). Different areas of CMBs play an important role in emotional control and executive function (17). In addition, damage to endothelial cells and blood-brain barrier have been reported to play an important role in CMBs, while vascular dysplasia might aggravate their pathological process. However, the underlying mechanisms of CMBs in depression are still unknown, although they might be 
explained by several pathways. Firstly, CMBs can cause structural disruptions of white matter tracts that are related to mood regulation and predispose an individual to depression (18). Secondly, microvascular dysfunction in subjects with CMBs is closely linked to chronic low-grade inflammation and/or oxidative stress (19). In fact, other studies have linked inflammation to depression or PSD via perfusion deficits or increased expression of serotonin receptors (20). Thirdly, CMBs might be associated with endothelial dysfunction, which could activate hemostatic mechanisms, inhibit reuptake of serotonin from the blood by platelets, reduce concentrations of intracellular serotonin, and eventually induce depression symptoms.

Serotonergic transmission deficiency is important in biological mechanisms during depression and PSD. Serotonergic innervations to the cerebral cortex and limbic structures originates almost exclusively from raphe nuclei in the caudal midbrain and rostral pons (21). Impaired serotonergic binding activities in these nuclei has been reported in PSD and major depression (22). In addition, structural disruptions in the brainstem raphe have consistently been found in depression and associated neuronal loss has further been reported in the same region (23). Meanwhile, deep brain CMBs, a kind of small cerebral vascular disease, reflect the disturbance of brain microcirculation with previous studies showing that chronic vascular burden might play an important role in PSD (24). Endothelial dysfunction and damage to the blood-brain barrier could lead to chronic hypoperfusion, which is related to chronic low-grade inflammation, and oxidative stress (19). Moreover, inflammation has been implicated in PSD through increased expression of serotonin receptors (20).

Current knowledge of microRNAs coupled with recent advancement in detection technology has increased research on miRNAs and stroke (25). Specifically, miRNAs are involved in and regulate many biological processes in the central nervous system, including endothelial dysfunction, apoptosis, cell proliferation, inflammatory response, oxidative stress, angiogenesis, and neurogenesis (26). Moreover, they are stable during blood circulation and have recently been identified as potential new types of biomarkers for treatment and diagnosis. Previous studies have shown that miRNAs play a role in the development of various mental disorders (27) with recent evidence implicating them in the occurrence, development, and treatment of depression (28).

In our previous study, we found a close relationship between TrkB and PSD (8). In the current study, we observed a correlation between miR-22 and TrkB gene (NTRK2) based on results from analysis of microRNAs target genes using an online prediction software. In addition, a significant differential expression was observed in miR-22 between patients with post-stroke and non-poststroke depression following gene chip analysis (9). All these studies suggest that miR-22 might be a potential biomarker for the diagnosis of PSD. In our study, we also found a significant increase in plasma miR-22 in subjects from the PSD relative to those in the non-PSD group, which confirmed the results of the gene chip. A further evaluation of the diagnostic value indicated that MiR-22 might be a new blood biomarker for PSD one month after stroke.

$B D N F$ has proven to be an important neurotrophic factor that affects neuron proliferation, synaptic function, and plasticity by binding to the receptor complex formed by TrkB (29). Activation of TrkB has been shown to result in phosphorylation of self-residues and sequential activation of intracellular cascade signals, including MAPK/ERK, $P L C \gamma$, and PI3K pathways (30). Phosphorylation of ERK indirectly promotes phosphorylation of $C R E B$ through its downstream signaling molecules. Phosphorylated $C R E B$ binds onto the promoter region of $B D N F$, initiating its transcription. The BDNF-ERK-CREB pathway plays an important role in various neurobiological processes such as synaptic plasticity (31). Damage resulting from neuronal plasticity has been proven to be important in PSD pathogenesis (32). Numerous studies have further shown that reduced $B D N F$ and TrkB are correlated to PSD $(8,33)$. miR-22 was reported to inhibit $B D N F$ expression by regulating translation of $B D N F$ mRNA at the post-transcriptional level. An elevated miR-22 expression was found to inhibit the BDNF-ERK-CREB pathway, leading to PSD (34). In this study, we found an association between miR-22 and TrkB genes (NTRK2) following online prediction. Our prediction indicated that miR-22 might bind onto the 3'UTR region of target mRNA transcribed by NTRK2, inhibit synthesis of target protein (TrkB) and associated signal cascade reaction, destroy neuron plasticity, and eventually lead to PSD.

Previous animal studies have shown that PSD-related glucocorticoid receptor (GR) decreased in the frontal cortex and hippocampus $(35,36)$. In addition, GR has been reported to inhibit expression of miR-22 by binding onto the transcription start site of miR-22 promoter (37). We hypothesized that PSD occurrence led to a decrease in GR and up-regulated expression of miR-22. Consequently, increased miR-22 expression could increase the risk of PSD, while occurrence of depression could also up-regulate miR-22 expression.

We also attempted to explore the possible mechanism of miR-22 action. Overall, we found that patients with brainstem and deep CMBs had a higher plasma miR-22 than the other group. This suggested that miR-22 was likely to be associated with brainstem and deep cerebral microvascular impairment, affecting the pathogenesis of PSD $(P=0.028)$. A significant statistical result might be found by increasing the sample size. Vascular endothelial cadherin (VE-cadherin) is an important adhesion molecule, and plays a key role in maintaining the integrity of endothelial barriers, inflammation, and regulation of angiogenesis (38). Previous studies have shown that miR-22 binds to the $3^{\prime} U T R$ region of VE-cadherin mRNA and regulates expression 
of VE-cadherin (10). Overexpression of miR-22 induced endothelial inflammation, damage to endothelial cells, increase in vascular permeability, destruction of bloodbrain barriers, and led to abnormal angiogenesis and vascular dysplasia. On the other hand, inhibition of miR22 expression protected vascular endothelial cells (10). Besides, damage to endothelial cells and blood-brain barriers played an important role in CMBs (39). Angiogenesis and myelin regeneration might maintain the stability and promote impairment of cerebral microvasculature through a coupling interaction (40). A high expression of miR-22 in PSD patients might lead to damage of endothelial cells, disrupt blood-brain barriers, interfere with endothelial-oligodendrocyte coupling, and cause impairment of cerebral microvasculature. It has further been shown that structural disruptions in the brainstem raphe and deep nerve pathways are accompanied by serotonergic transmission deficiency, which is eventually involved in the pathophysiology of PSD (23).

\section{References}

1. Ayerbe L, Ayis S, Crichton S, Wolfe CD, Rudd AG. The long-term outcomes of depression up to 10 years after stroke; the south london stroke register. J Neurol Neurosurg Psychiatry 2014; 85: 514-521, doi: 10.1136/jnnp2013-306448

2. Fabian MR, Sonenberg N, Filipowicz W. Regulation of mrna translation and stability by micrornas. Annu Rev Biochem 2010; 79: 351-379, doi: 10.1146/annurev-biochem-060308103103.

3. Jiang $X G$, Lin $Y, L i$ YS. Correlative study on risk factors of depression among acute stroke patients. Eur Rev Med Pharmacol Sci 2014; 18: 1315-1323.

4. Serafini G, Pompili M, Hansen KF, Obrietan K, Dwivedi Y, Shomron N, Girardi P. The involvement of micrornas in major depression, suicidal behavior, and related disorders: a focus on mir-185 and mir-491-3p. Cell Mol Neurobiol 2014; 34: 17-30, doi: 10.1007/s10571-013-9997-5.

5. Roy B, Dunbar M, Shelton RC, Dwivedi Y. Identification of microrna-124-3p as a putative epigenetic signature of major depressive disorder. Neuropsychopharmacology 2017;42: 864-875, doi: 10.1038/npp.2016.175.

6. Reinhart V, Bove SE, Volfson D, Lewis DA, Kleiman RJ, Lanz TA. Evaluation of trkb and bdnf transcripts in prefrontal cortex, hippocampus, and striatum from subjects with schizophrenia, bipolar disorder, and major depressive disorder. Neurobiol Dis 2015; 77: 220-227, doi: 10.1016/j.nbd. 2015.03.011.

7. Jiang H, Chen S, Li C, Lu N, Yue Y, Yin Y, et al. The serum protein levels of the tpa-bdnf pathway are implicated in depression and antidepressant treatment. Trans/ Psychiatry 2017; 7: e1079, doi: 10.1038/tp.2017.43.

8. Zhou Z, Ding X, Yang Q, Hu J, Shang X, Huang X, et al. Association between single-nucleotide polymorphisms of the tyrosine kinase receptor $\mathrm{b}$ (trkb) and post-stroke depression in china. PloS One 2015; 10: e0144301, doi: 10.1371/ journal.pone. 0144301 .
A number of limitations in our study are as follows: firstly, this was a single-center retrospective study with a limited sample size. It will be necessary to confirm these findings using large prospective clinical studies. Secondly, some patients with severe aphasia and severe disease were excluded from the study, which may introduce some bias in the observed results. Finally, during the 1-month period between MRI examination and depression evaluation, additional vascular events or other clinical events might have occurred, which could have affected the diagnosis of PSD.

\section{Acknowledgments}

The authors are sincerely grateful to all study participants. This study was supported by grants from Wannan Medical College Young and Middle-Aged Natural Science Foundation.

9. Zhang Y, Cheng L, Chen Y, Yang GY, Liu J, Zeng L. Clinical predictor and circulating microrna profile expression in patients with early onset post-stroke depression. J Affect Disord 2016; 193: 51-58, doi: 10.1016/j.jad.2015.12.061.

10. Gu W, Zhan H, Zhou XY, Yao L, Yan M, Chen A, et al. Microrna-22 regulates inflammation and angiogenesis via targeting ve-cadherin. FEBS Lett 2017; 591: 513-526, doi: 10.1002/1873-3468.12565.

11. Direk N, Perez HS, Akoudad S, Verhaaren BF, Niessen WJ, Hofman A, et al. Markers of cerebral small vessel disease and severity of depression in the general population. Psychiatry Res Neuroimaging 2016; 253: 1-6, doi: 10.1016/ j.pscychresns.2016.05.002.

12. Adams HP Jr, Bendixen BH, Kappelle LJ, Biller J, Love BB, Gordon DL, et al. Classification of subtype of acute ischemic stroke. Definitions for use in a multicenter clinical trial. TOAST. Trial of org 10172 in acute stroke treatment. Stroke 1993; 24: 35-41, doi: 10.1161/01.STR.24.1.35.

13. Hamilton M. A rating scale for depression. J Neurol Neurosurg Psychiatry 1960; 23: 56-62, doi: 10.1136/jnnp.23.1.56.

14. Tang DY, Liu AC, Leung MH, Siu BW. Antisocial personality disorder subscale (chinese version) of the structured clinical interview for the dsm-iv axis ii disorders: Validation study in cantonese-speaking hong kong chinese. East Asian Arch Psychiatry 2013; 23: 37-44.

15. Wardlaw JM, Smith EE, Biessels GJ, Cordonnier C, Fazekas F, Frayne R, et al. Neuroimaging standards for research into small vessel disease and its contribution to ageing and neurodegeneration. Lancet Neurol 2013; 12: 822-838, doi: 10.1016/S1474-4422(13)70124-8.

16. Livak KJ, Schmittgen TD. Analysis of relative gene expression data using real-time quantitative pcr and the 2(-delta delta c(t)) method. Methods 2001; 25: 402-408, doi: 10.1006/meth.2001.1262.

17. Becker G, Berg D, Lesch KP, Becker T. Basal limbic system alteration in major depression: a hypothesis supported by 
transcranial sonography and mri findings. Int J Neuropsychopharmacol 2001; 4: 21-31, doi: $10.1017 /$ S1461145701 002164.

18. Fazekas F, Kleinert R, Roob G, Kleinert G, Kapeller P, Schmidt $R$, et al. Histopathologic analysis of foci of signal loss on gradient-echo $\mathrm{t} 2{ }^{*}$-weighted $\mathrm{mr}$ images in patients with spontaneous intracerebral hemorrhage: evidence of microangiopathy-related microbleeds. AJNR Am J Neuroradiol 1999; 20: 637-642.

19. van Dooren FE, Schram MT, Schalkwijk CG, Stehouwer CD, Henry RM, Dagnelie PC, et al. Associations of low-grade inflammation and endothelial dysfunction with depression the maastricht study. Brain Behav Immun 2016; 56: 390396, doi: 10.1016/j.bbi.2016.03.004.

20. Buga AM, Ciobanu O, Badescu GM, Bogdan C, Weston R, Slevin $\mathrm{M}$, et al. Up-regulation of serotonin receptor $2 \mathrm{~b}$ mrna and protein in the peri-infarcted area of aged rats and stroke patients. Oncotarget 2016; 7: 17415-17430, doi: 10.18632/ oncotarget.8277.

21. Miller JM, Hesselgrave N, Ogden RT, Sullivan GM, Oquendo MA, Mann JJ, et al. Positron emission tomography quantification of serotonin transporter in suicide attempters with major depressive disorder. Biol Psychiatry 2013; 74: 287295, doi: 10.1016/j.biopsych.2013.01.024.

22. Lowry CA, Hale MW, Evans AK, Heerkens J, Staub DR, Gasser PJ, et al. Serotonergic systems, anxiety, and affective disorder: Focus on the dorsomedial part of the dorsal raphe nucleus. Ann N Y Acad Sci 2008; 1148: 86-94, doi: 10.1196/annals.1410.004.

23. Abe O, Yamasue $H$, Kasai K, Yamada H, Aoki S, Inoue H, et al. Voxel-based analyses of gray/white matter volume and diffusion tensor data in major depression. Psychiatry Res 2010; 181: 64-70, doi: 10.1016/j.pscychresns.2009.07.007.

24. Romero JR, Preis SR, Beiser A, Himali JJ, Shoamanesh A, Wolf PA, et al. Cerebral microbleeds as predictors of mortality: the Framingham heart study. Stroke 2017; 48: 781-783, doi: 10.1161/STROKEAHA.116.015354.

25. Mirzaei H, Momeni F, Saadatpour L, Sahebkar A, Goodarzi M, Masoudifar A, et al. Microrna: relevance to stroke diagnosis, prognosis, and therapy. J Cell Physiol 2018; 233: 856-865, doi: 10.1002/jcp.25787.

26. Abernathy DG, Yoo AS. Microrna-dependent genetic networks during neural development. Cell Tissue Res 2015; 359: 179-185, doi: 10.1007/s00441-014-1899-4.

27. Luoni A, Riva MA. Micrornas and psychiatric disorders: from aetiology to treatment. Pharmacol Ther 2016;167: 13-27, doi: 10.1016/j.pharmthera.2016.07.006.

28. Lopez JP, Kos A, Turecki G. Major depression and its treatment: Micrornas as peripheral biomarkers of diagnosis and treatment response. Curr Opin Psychiatry 2018; 31: 7-16, doi: 10.1097/YCO.0000000000000379.

29. Zhang JC, Yao W, Hashimoto K. Brain-derived neurotrophic factor (bdnf)-trkb signaling in inflammation-related depression and potential therapeutic targets. Curr Neuropharmacol 2016; 14: 721-731, doi: 10.2174/1570159X14666160119 094646.
30. Liang J, Deng G, Huang $\mathrm{H}$. The activation of bdnf reduced inflammation in a spinal cord injury model by trkb/p38 mapk signaling. Exp Ther Med 2019; 17: 1688-1696, doi: 10.3892/etm.2018.7109.

31. Dwivedi Y, Rizavi HS, Roberts RC, Conley RC, Tamminga CA, Pandey GN. Reduced activation and expression of erk $1 / 2$ map kinase in the post-mortem brain of depressed suicide subjects. J Neurochem 2001; 77: 916-928, doi: 10.1046/j.1471-4159.2001.00300.x.

32. Tarai S, Mukherjee R, Gupta S, Rizvanov AA, Palotas A, Chandrasekhar Pammi VS, et al. Influence of pharmacological and epigenetic factors to suppress neurotrophic factors and enhance neural plasticity in stress and mood disorders. Cogn Neurodyn 2019; 13: 219-237, doi: 10.1007/s11571019-09522-3.

33. Chen MH, Lin WC, Wu HJ, Cheng CM, Li CT, Hong CJ, et al. Antisuicidal effect, BDNF Val66Met polymorphism, and lowdose ketamine infusion: reanalysis of adjunctive ketamine study of Taiwanese patients with treatment-resistant depression (AKSTP-TRD). J Affect Disord 2019; 251: 162-169, doi: 10.1016/j.jad.2019.03.075.

34. Muinos-Gimeno M, Espinosa-Parrilla Y, Guidi M, Kagerbauer B, Sipila T, Maron E, et al. Human micrornas miR-22, miR-138-2, miR-148a, and miR-488 are associated with panic disorder and regulate several anxiety candidate genes and related pathways. Biol Psychiatry 2011; 69: 526-533, doi: 10.1016/j.biopsych.2010.10.010.

35. de la Tremblaye PB, Raymond J, Milot MR, Merali Z, Plamondon $\mathrm{H}$. Evidence of lasting dysregulation of neuroendocrine and hpa axis function following global cerebral ischemia in male rats and the effect of antalarmin on plasma corticosterone level. Horm Behav 2014; 65: 273-284, doi: 10.1016/j.yhbeh.2014.01.003.

36. Mamalaki E, Kvetnansky R, Brady LS, Gold PW, Herkenham M. Repeated immobilization stress alters tyrosine hydroxylase, corticotropin-releasing hormone and corticosteroid receptor messenger ribonucleic acid levels in rat brain. J Neuroendocrinol 1992; 4: 689-699, doi: 10.1111/ j.1365-2826.1992.tb00220.x.

37. Fu Q, Liu CJ, Zhang X, Zhai ZS, Wang YZ, Hu MX, et al. Glucocorticoid receptor regulates expression of microrna-22 and downstream signaling pathway in apoptosis of pancreatic acinar cells. World J Gastroenterol 2018; 24: 51205130, doi: 10.3748/wjg.v24.i45.5120.

38. Muller WA. The regulation of transendothelial migration: New knowledge and new questions. Cardiovasc Res 2015; 107: 310-320, doi: 10.1093/cvr/cvv145.

39. Cuadrado-Godia E, Dwivedi P, Sharma S, Ois Santiago A, Roquer Gonzalez J, Balcells M, et al. Cerebral small vessel disease: A review focusing on pathophysiology, biomarkers, and machine learning strategies. J Stroke 2018; 20: 302320, doi: 10.5853/jos.2017.02922.

40. Miyamoto N, Pham LD, Seo JH, Kim KW, Lo EH, Arai K. Crosstalk between cerebral endothelium and oligodendrocyte. Cell Mol Life Sci 2014; 71: 1055-1066, doi: 10.1007/ s00018-013-1488-9. 